\title{
Tissue-Engineered Tendon for Enthesis Regeneration in a Rat Rotator Cuff Model
}

\author{
Michael J. Smietana, Pablo Moncada-Larrotiz, ${ }^{2}$ Ellen M. Arruda, ${ }^{1,3,4}$ Asheesh Bedi, ${ }^{5}$ and Lisa M. Larkin ${ }^{1,2, *}$
}

\begin{abstract}
Healing of rotator cuff (RC) injuries with current suture or augmented scaffold techniques fails to regenerate the enthesis and instead forms a weaker fibrovascular scar that is prone to subsequent failure. Regeneration of the enthesis is the key to improving clinical outcomes for RC injuries. We hypothesized that the utilization of our tissue-engineered tendon to repair either an acute or a chronic full-thickness supraspinatus tear would regenerate a functional enthesis and return the biomechanics of the tendon back to that found in native tissue. Engineered tendons were fabricated from bone marrow-derived mesenchymal stem cells utilizing our well-described fabrication technology. Forty-three rats underwent unilateral detachment of the supraspinatus tendon followed by acute (immediate) or chronic (4 weeks retracted) repair by using either our engineered tendon or a transosseous suture technique. Animals were sacrificed at 8 weeks. Biomechanical and histological analyses of the regenerated enthesis and tendon were performed. Statistical analysis was performed by using a one-way analysis of variance with significance set at $p<0.05$. Acute repairs using engineered tendon had improved enthesis structure and lower biomechanical failures compared with suture repairs. Chronic repairs with engineered tendon had a more native-like enthesis with increased fibrocartilage formation, reduced scar formation, and lower biomechanical failure compared with suture repair. Thus, the utilization of our tissue-engineered tendon showed improve enthesis regeneration and improved function in chronic RC repairs compared with suture repair. Clinical Significance: Our engineered tendon construct shows promise as a clinically relevant method for repair of RC injuries.
\end{abstract}

Keywords: enthesis, rotator cuff; scaffold-less, shoulder; tendon-to-bone; tissue engineering

\section{Introduction}

Rotator cuff (RC) tears are one of the most common orthopedic disorders in the United States, with more than 200,000 repair procedures performed annually. ${ }^{1}$ Tears of the RC tendons often fail to heal without intervention and are frequently the cause of significant shoulder pain and impaired shoulder function. ${ }^{2}$ Current repair techniques utilize an open or arthroscopic suture technique to reattach the tendon to bone. Failure rates, however, especially in full-thickness large (3$5 \mathrm{~cm}$ ) or massive tears $(>5 \mathrm{~cm}, 2$ tendons), are unac- ceptably high (20-94\%). ${ }^{2,3}$ Factors including tendon retraction, muscle atrophy, and fatty infiltration have been known to limit the successfulness of the repair. ${ }^{4-7}$ In addition, healing of the enthesis with current techniques often results in a notably weaker fibrovascular scar rather than a normal enthesis architecture and may explain the high prevalence of repair failures. ${ }^{8}$

The native enthesis consists of a compositionally and mechanically graded fibrocartilage transition zone that is designed to minimize stress concentrations at the tendon-bone interface. Using a rat supraspinatus RC

Departments of ${ }^{1}$ Biomedical Engineering, ${ }^{2}$ Molecular \& Integrative Physiology, ${ }^{3}$ Mechanical Engineering, ${ }^{4}$ Macromolecular Science and Engineering, and ${ }^{5}$ Orthopedic Surgery, University of Michigan, Ann Arbor, Michigan.

*Address correspondence to: Lisa Larkin, PhD, Molecular and Integrative Physiology, University of Michigan, Biomedical Science Research Building (BSRB), 109 Zina Pitcher Place, Room \#2025, Ann Arbor, MI 48109-2200, E-mail: llarkin@umich.edu

(c) Michael J. Smietana et al. 2017; Published by Mary Ann Liebert, Inc. This is an Open Access article distributed under the terms of the Creative Commons Attribution License, which permits unrestricted use, distribution, and reproduction in any medium, provided the original work is properly cited. 
model, Thomopoulos et al. demonstrated that the biology and zonal arrangement of the enthesis is not regenerated after RC repair. ${ }^{9}$ Rather, a sharp boundary was observed, with disorganized collagen fibers and a fibrocartilaginous region, representing a significant deviation from the native enthesis anatomy. The regeneration of a structurally sound enthesis with normal biomechanics is paramount for improving functional outcomes and reducing the risk of recurrent structural failure after the repair of RC injuries. As such, several tissue-engineering approaches are being developed to improve the regeneration of the enthesis after the repair of RC tears.

To date, there are several commercially available extracellular matrix (ECM) scaffolds being tested clinically to improve enthesis repair after RC repair. ${ }^{10}$ Although the biochemical properties of these scaffold materials have the potential to modulate host response and influence enthesis repair, the structural properties degrade over time and often do not match the rate of new ECM regeneration. In addition, these ECM scaffolds often fail to regenerate the fibrocartilage gradient at the enthesis, leading to stress points and weaker connections to the bone. Thus, alternative strategies are needed to improve enthesis regeneration after RC injuries.

Recently, stratified and functionally graded scaffoldbased approaches have been developed to engineer the native tendon-to-bone interface in vitro. ${ }^{11,12}$ In these studies, the spatial distribution of mechanical properties, biological factors, and/or cell populations are used to direct the formation of gradients that are designed to mimic the transition of tendon to bone (enthesis) in vitro or after implantation into an ectopic site. ${ }^{13}$ Though a promising idea, the evaluation of these graded scaffolds in an in vivo RC repair model has not been performed. Thus, the degradation characteristics of these gradient scaffolds and their biocompatibility have not yet been defined.

To address the concerns of scaffold-based approaches, our lab has developed a scaffold-less tissueengineered tendon construct that relies on in vivo biologic and mechanical environments to direct the regeneration of the tendon and enthesis. Our previous work in sheep anterior cruciate ligament (ACL) and rat medial collateral ligament repair models has shown that the osteogenic regions of our construct quickly integrate with the host boney tissue and rapidly remodel, forming a graded tendon or ligamentto-bone transition and a functional enthesis that remodels in vivo to match the native footprint. ${ }^{14,15}$
Although the results of these studies are encouraging, it is unclear as to whether we can extrapolate these findings to an RC injury model. Thus, the objective of this study was to evaluate the efficacy of our scaffold-less tendon constructs to regenerate the native-like structure of tendon and the mechanical strength of the enthesis in both acute (immediate repair) and chronic (repair 4 weeks post-injury) supraspinatus tears in a rat model. We hypothesized that utilization of the tissue-engineered tendon construct would better regenerate the fibrocartilage transition zone, collagen organization, and tendon attachment strength of the native enthesis in both acute and chronic tear repairs compared with suture repair alone.

\section{Materials and Methods}

Animal model and design

Female Fisher 344 rats, approximately $150-200 \mathrm{~g}$ in mass, were obtained from Charles River Laboratories, Inc. All animals were acclimated to colony conditions for 1 week before any procedure. Animals were fed Purina Rodent Chow 5001 and water ad libitum. All surgical procedures were performed in an aseptic environment, with animals in a deep plane of anesthesia induced by intraperitoneal injections of sodium pentobarbital $(65 \mathrm{mg} / \mathrm{kg})$. Supplemental doses of pentobarbital were administered as required to maintain an adequate depth of anesthesia. All animal care and animal surgery procedures were in accordance with The Guide for Care and Use of Laboratory Animals, ${ }^{29}$ and the protocol was approved by the University of Michigan's, University Committee for the Use and Care of Animals.

For recreating an acute supraspinatus tendon tear, the supraspinatus tendons of the right shoulders underwent a full-thickness tenectomy from the humerus by using a transacromial approach ${ }^{16}$ and were then immediately repaired with either a Mason-Allen suture technique (suture repair group) or a modified transosseous technique utilizing a tissue-engineered tendon construct (construct repair group). Chronic tears of the supraspinatus tendon were created by severing the supraspinatus tendon from the humerus and then encasing the detached end of the tendon in silicone tubing. The animals were then allowed to recover for 4 weeks with the detached tendon. The encasement with the silicone tubing prevented attachment to the surrounding connective tissue so that the cut end of the tendon could easily be identified and repaired at a later date. The contralateral shoulders were used as 
non-surgical controls for each treatment group. Next, the chronic tendon tears were repaired with either a Mason-Allen suture technique (suture repair group) or a modified trans-osseous technique utilizing a tissue-engineered tendon construct (construct repair group). At 8 weeks after repair of the tendon, animals were euthanized $(n=43)$ with an overdose of sodium pentobarbital and at least five specimens per treatment group were assessed for biomechanical and structural regeneration of the injured tendon, respectively.

\section{Preparation of solutions and media}

Media were prepared, as previously described, with slight modifications. ${ }^{15,17}$ Growth medium (GM) consisted of Dulbecco's modified Eagle's medium (DMEM; Gibco, Cat\# 10565-042) supplemented with 20\% Fetal Bovine Serum (FBS; Gibco, Cat\# 10437-028), 2\% antibiotic-antimycotic (Sigma; Cat\# A9909), $600 \mathrm{ng} / \mathrm{mL}$ $\mathrm{bFGF}$, and $40 \mathrm{ng} / \mathrm{mL}$ dexamethasone. Differentiation medium (DM) consisted of DMEM supplemented with 7\% Horse Serum Albumin (HS; Gibco, Cat\# 16050122 ) and $2 \%$ antibiotic-antimycotic, plus the addition of $0.13 \mathrm{mg} / \mathrm{mL}$ ascorbic acid-2 phosphate, $0.05 \mathrm{mg} / \mathrm{mL}$ L-proline, $400 \mathrm{ng} / \mathrm{mL}$ dexamethasone, and $2 \mathrm{ng} / \mathrm{mL}$ transforming growth factor-beta (TGF- $\beta$; Peprotech, Cat\# 100-21).

\section{Bone marrow isolation and cell expansion}

Bone marrow stromal cells were isolated and expanded according to a previously described protocol. ${ }^{15,17}$ Briefly, rat femora were removed from the animal and cut at the proximal and distal metaphysis. The marrow contents were extracted through centrifugation $(900 \mathrm{~g}$ for $10 \mathrm{~min}$ ) and cultured on $100 \mathrm{~mm}$ tissue-culture-treated polystyrene plates (BD Falcon). Plates were fed GM and incubated at $37^{\circ} \mathrm{C}, 95 \%$ humidity, and $5 \% \mathrm{CO}_{2}$. After $72 \mathrm{~h}$, plates were rinsed with DPBS and fed with fresh GM, removing the non-adherent cells. The remaining adherent cell population was cultured to $80 \%$ confluence, at which time cells were enzymatically removed from the $100 \mathrm{~mm}$ plate by using a $0.25 \%$ trypsin-EDTA solution (Gibco), passaged, and seeded onto $100 \mathrm{~mm}$ plates at a density of $5 \times 10^{3}$ cells $/ \mathrm{cm}^{2}$. After 3-4 passages, the cultured cells were utilized for construct fabrication.

Preparation of self-organized tendon constructs Methods for creating scaffold-less three-dimensional tendon/ligament tissues have been previously described. ${ }^{14,15,17}$ Briefly, mesenchymal stem cells (MSCs) were plated onto $100 \mathrm{~mm}$ cell culture dishes at $21 \times 10^{3}$ cells $/ \mathrm{cm}^{2}$ and suspended in $8 \mathrm{~mL}$ of GM supplemented with $0.13 \mathrm{mg} / \mathrm{mL}$ ascorbic acid-2 phosphate and $0.05 \mathrm{mg} / \mathrm{mL}$ L-proline. The dishes were placed in a $37^{\circ} \mathrm{C}, 5 \% \mathrm{CO}_{2}$ incubator and medium was changed every 2-3 days. After approximately 5 days, the cells became confluent and DM was substituted for GM to induce monolayer delamination. As the monolayer delaminated, it was transferred to a Sylgard coated dish and constrained between two minutien pins that were placed approximately $60 \mathrm{~mm}$ apart. The monolayer was fully self-assembled into a $60 \mathrm{~mm}$-long cylindrical construct within 1-2 days. One week after delamination, constructs that were approximately $0.9 \mathrm{~mm}$ in diameter were cut into $6 \mathrm{~mm}$-long segments for use in RC repairs. The medium was again changed every 2-3 days until the construct was used for implantation.

\section{Surgical technique}

Rats were anesthetized by using $2 \%$ isoflurane, and the right shoulder was shaved and scrubbed with povidone iodine. An incision was made from a point $2-3 \mathrm{~mm}$ distal to the head on the humerus to approximately $5 \mathrm{~mm}$ proximal along the deltoid muscle. The deltoid muscle was cut longitudinally to expose the supraspinatus muscle. The acromion was cut and moved to expose the enthesis of the supraspinatus on the humeral head. The supraspinatus was isolated, and the tendon was sharply released from the bone at the enthesis. For acute suture repairs, two $0.6 \mathrm{~mm}$ crossing tunnels were drilled into the greater tuberosity footprint. A 4-0 Vicryl suture (Ethilon) was used to suture the detached supraspinatus tendon back onto its original insertion point. For the acute construct repairs, a $0.9 \mathrm{~mm}$ bone tunnel was drilled directly through the middle of supraspinatus insertion footprint and guided out to the lateral aspect of the humeral head. A tissue-engineered graft ( $\sim 6 \mathrm{~mm}$ in length) was pulled into the bone tunnel and anchored to the periosteum on the lateral aspect of the humerus with 9-0 silk sutures (Ethilon) (Fig. 1).

A small hole was made in the remaining supraspinatus tendon with \#5 forceps and the construct was pulled into the hole, such that the graft lay on the posterior aspect of the tendon. The end of the construct was sutured to the supraspinatus tendon with $9-0$ silk sutures. The deltoid and biceps muscle were closed by using 7-0 sutures, and the skin was closed with staples. Animals were fed ad libitum and allowed full cage activity. Analgesic, Carprofen ( $5 \mathrm{mg} / \mathrm{kg})$, was administered 


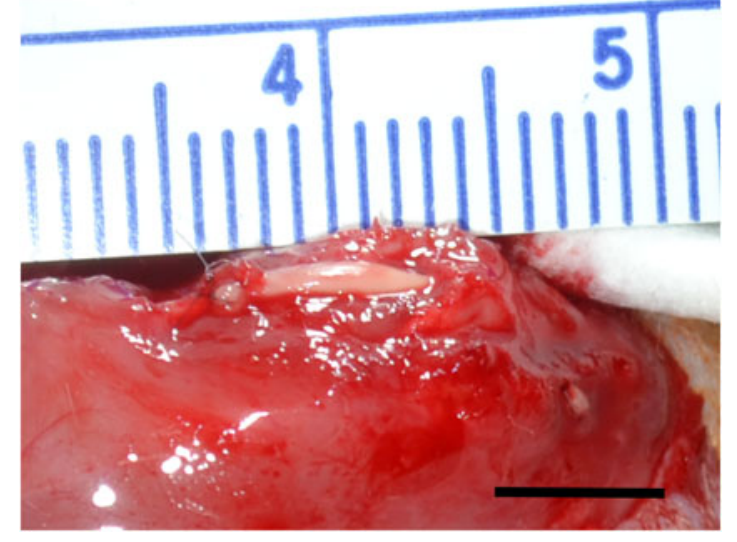

FIG. 1. Tissue-engineered construct inserted into humeral bone tunnel during repair of acute rotator cuff tear. Scale bar represents $5 \mathrm{~mm}$.

after induction of anesthesia and every $24 \mathrm{~h}$ for $48 \mathrm{~h}$ post-surgery. For the chronic groups, the same surgical repairs were performed after 4 weeks of chronic tendon detachment. Eight weeks after surgical repair of the tendon, the animals were euthanized with sodium pentobarbital and both shoulders were removed for biomechanical and histological analyses.

\section{Biomechanical testing}

Biomechanical testing was performed on freshly dissected supraspinatus tendons while they were still attached to the humerus. Before testing, the supraspinatus muscle belly was carefully removed from the supraspinatus tendon and the cross-sectional area of the tendon was measured by using a digital micrometer. To prevent the fracture of the humeral physis during mechanical testing, the humerus was potted with polymer into a custom-designed grip. The specimen was then placed into a Dynamic Mechanical Analyzer (RSA-III; TA Instruments) and aligned in the native joint flexion angle for uniaxial tensile testing. The end of the tendon was secured to a screw grip by using sandpaper and cyanoacrylate. The specimen was attached to a $35 \mathrm{~N}$ load cell and aligned uniaxially. The specimen was loaded to failure at a rate of $0.3 \mathrm{~mm} /$ sec or approximately $0.10 / \mathrm{sec}$. Samples were tested at room temperature and were continuously moistened via a PBS drip throughout testing. The ultimate load to failure and location of failure (enthesis or tendon mid-substance) were recorded. The stiffness for each specimen was calculated from the linear region of the load-deformation curve. Statistical analysis was performed by two-way analysis of variance (ANOVA), with significance as $p<0.05$. Data from the contralateral shoulders were combined for each injury group. Data are presented as mean \pm standard error.

\section{Histomorphometric analysis}

After recovery, the supraspinatus tendon with the humerus still attached was carefully dissected from the surrounding tissue under a microscope, leaving the supraspinatus muscle intact. After dissection, samples were fixed in $10 \%$ neutral buffered formalin for $48 \mathrm{~h}$, decalcified in EDTA for $48 \mathrm{~h}$, and, subsequently, washed in phosphate-buffered saline solution. The samples were dehydrated and embedded in paraffin for sectioning. To facilitate comparisons between groups, an orientation of $90^{\circ}$ between the humerus and supraspinatus muscle was maintained for each sample during embedding. Serial sections that were $12-\mu \mathrm{m}$ thick, containing the repaired tendon and greater tuberosity of the humerus, were cut in the coronal plane and stained with picosirius red, safranin-O, and hematoxylin and eosin (H\&E). Using an Olympus B light microscope, digital images of the stained slides were taken by using a SPOT RT camera (Diagnostic Instruments). Five representative slides for each stain and specimen were prepared. Each slide was evaluated by using polarized and non-polarized light and analyzed by three different people.

To evaluate collagen organization in the repaired tendon enthesis, sections stained with picrosirius red were imaged under monochromatic polarized light. The birefringence of collagen fibers was semi-quantitatively analyzed, based on brightness, to determine differences in collagen deposition and maturation at the site of repair. ${ }^{18,19}$ Five representative tissue sections across the full width of the tendon-bone interface were analyzed for each shoulder. The polarization plane was rotated until maximum brightness was obtained to account for variations in specimen orientation on the slide. Digital images were taken of each tissue section at $\times 10$ magnification during a single imaging session under identical imaging parameters. The images were imported into Image J software and underwent 8-bit digitalization. Non-collagenous material was shown as black (zero), and collagenous material was assigned a gray-scale value from 1 to 255 . Higher gray-scale values signify more organized and mature collagen. Using the Image J software, ten rectangular areas $(50 \times 50 \mu \mathrm{m})$ were randomly selected in the tendon directly adjacent to the 
tendon-bone interface. Mean gray-scale values for each region were averaged for each specimen to obtain a brightness value. Statistical analysis of specimen brightness was performed by two-way ANOVA, with $p$ value $<0.05$ considered significant. Data are presented as mean \pm standard error.

The area of fibrocartilaginous material at the tendonbone interface was assessed by use of safranin O-stained sections. Safranin O stains sulfated proteoglycans reddishpurple, a property referred to as metachromasia. Digital images of the stained sections were imported into Image $\mathrm{J}$, and areas of metachromasia were meticulously outlined and measured by using the Image $J$ software at a $\times 4$ magnification (National Institutes of Health). Measured areas were averaged for suture- and construct-repaired shoulders and contralateral controls. Statistical analysis of the total area of fibrocartilage was performed by two-way ANOVA, with $p$ value $<0.05$ considered significant. Data are presented as mean \pm standard error.

\section{Results}

\section{Gross morphology}

At the time of necropsy, there was continuity between the tendon and bone in all of the acute and chronic repairs, with no detectable differences between groups. All animals appeared healthy and showed no signs of infection, or a negative immune or inflammatory response to the implanted graft. The cross-sectional area of the enthesis was measured and was not significantly different between the acute and chronic injury repairs $\left(3.4 \pm 0.8 \mathrm{~mm}^{2}\right)$.

\section{Biomechanical testing}

A minimum of five samples for each treatment group was tested uniaxially until failure. In the acute injury repair groups, four of the suture-repaired specimens $(n=8)$ failed at the enthesis, whereas the rest of the suture-repaired specimens and all the constructrepaired specimens $(n=5)$ failed at the tendon midsubstance. In the chronic injury repair group, three suture-repaired specimens $(n=5)$ failed at the enthesis whereas the rest of the suture-repaired specimens and the entire construct repair group $(n=5)$ failed at the tendon mid-substance. All non-operative contralateral shoulders failed at the tendon midsubstance during testing. Thus, the construct repair group was more similar in failure mode to the control than the suture-repaired group.

After 8 weeks of recovery, acute and chronic injuries repaired with either suture or construct techniques displayed no differences in stiffness between groups. All repair groups were significantly less stiff than their respective contralateral shoulders and not significantly different from each other (Fig. 2A). The mean stiffness of the tendon-bone interface in the acute injury repair groups was as follows: contra-lateral $(23.2 \pm 1.5 \mathrm{~N} / \mathrm{mm}$,
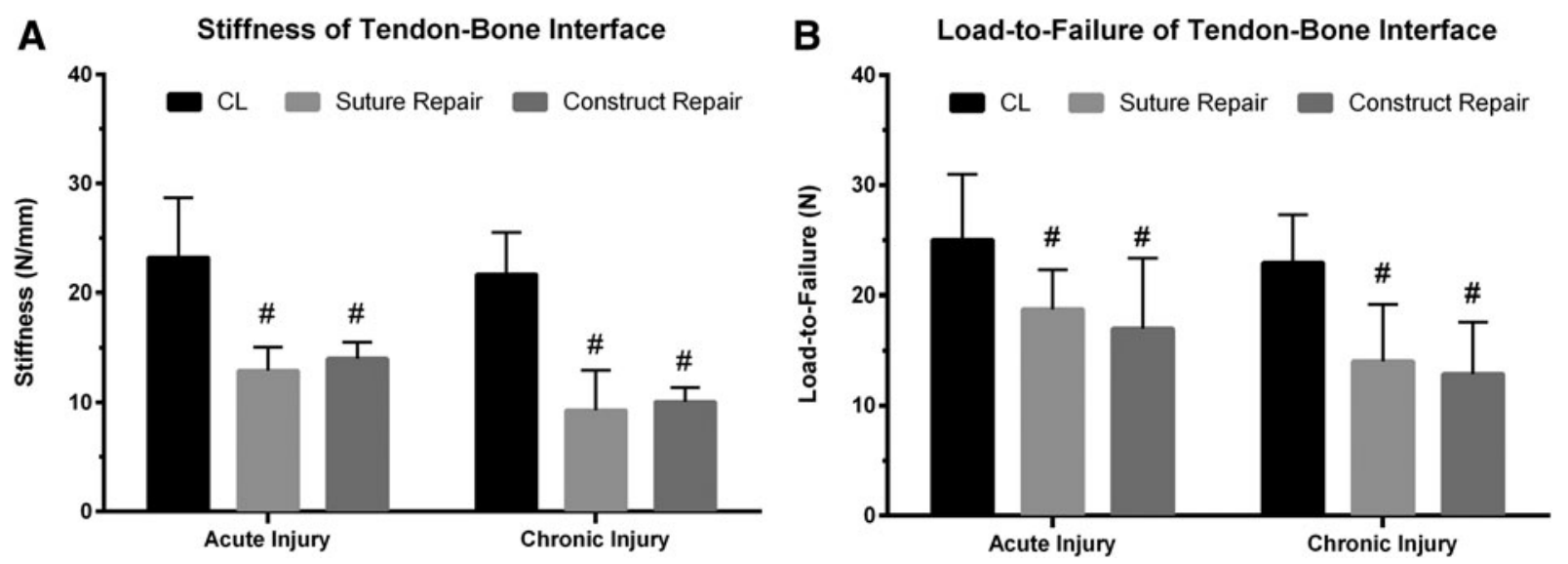

FIG. 2. Mechanics of tendon-bone interface after acute and chronic injury repair. (A) Stiffness of CL, suture-repaired, and construct-repaired shoulders and (B) load to failure of CL, suture-repaired, and construct-repaired shoulders. (\#) Denotes statistical significance compared with CL shoulder $(p<0.05)$. Data are shown as mean $\pm \mathrm{SE}$ for each group. $\mathrm{CL}$, contralateral. 
$n=13)$, suture repair $(12.9 \pm 0.8 \mathrm{~N} / \mathrm{mm}, n=8)$, and construct repair $(14.0 \pm 0.7 \mathrm{~N} / \mathrm{mm}, n=5)$. The sutureand construct-repaired shoulders achieved $56 \%$ and $60 \%$ of native contralateral stiffness, respectively. The mean stiffness of the tendon-bone interface in the chronic injury repair groups was as follows: contra-lateral $(21.7 \pm 1.2 \mathrm{~N} / \mathrm{mm}, n=10)$, suture repair $(9.3 \pm 1.6 \mathrm{~N} / \mathrm{mm}$, $n=5)$, and construct repair $(10.1 \pm 0.6 \mathrm{~N} / \mathrm{mm}, n=5)$. The suture- and construct-repaired shoulders achieved $43 \%$ and $46 \%$ of native contralateral stiffness, respectively.

The maximum load to failure was not significantly different between the acute and chronic repair groups with either suture or engineered tendon constructs. Each group was significantly less than its respective non-operative contralateral shoulder and not significantly different from the other repair groups (Fig. 2B). The maximum load to failure for acute injury repair was as follows: contra-lateral $(25.0 \pm 1.7 \mathrm{~N}$, $n=13)$, suture repair $(18.8 \pm 1.3 \mathrm{~N}, n=8)$, and construct repair $(17.0 \pm 2.8 \mathrm{~N}, n=5)$. This corresponded to approximately $75 \%$ and $68 \%$ of the contralateral failure load for suture and construct repairs, respectively. The maximum load to failure for chronic injury repair groups was as follows: contra-lateral (22.9 \pm $1.4 \mathrm{~N}, n=10)$, suture repair $(14.0 \pm 2.3 \mathrm{~N}, n=5)$, and construct repair $(12.9 \pm 2.1 \mathrm{~N}, n=5)$, respectively. The suture- and construct-repaired shoulders achieved $61 \%$ and $56 \%$ of contralateral shoulder load to failure, respectively.

\section{Histomorphometric analysis}

Collagen birefringence was semi-quantitatively analyzed, based on brightness, to evaluate collagen organization at the tendon-bone interface. Five specimens were analyzed for each treatment group. Contralateral shoulders of acute and chronic injured shoulders were not significantly different and were combined for analysis. Suture- and construct-repaired shoulders, although significantly more disorganized (shown in picrosirius red staining) than contralateral shoulders, were not significantly different from each other in either acute or chronic injury groups (Fig. 3). In addition, although repaired chronic injuries trended toward lower grayscale values, no significant difference in collagen organization was observed between acutely and chronically injured shoulders. Suture repairs of acute injuries had a mean brightness gray-scale value of $70 \pm 1$ gray-scale units, whereas construct repairs had a gray-scale value of $70 \pm 4$ gray-scale units. Chronic injuries repaired

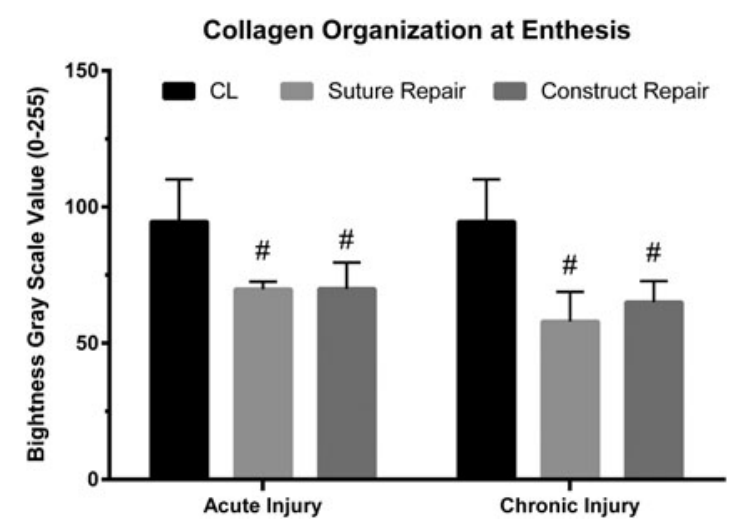

FIG. 3. Collagen organization at enthesis after acute and chronic injury repair. No significant differences were observed between acute and chronic injury groups. (\#) Denotes statistical significance compared with $\mathrm{CL}$ shoulder $(p<0.05)$. Data are shown as mean \pm SE for each group.

with suture and construct techniques had gray-scale values of $58 \pm 5$ and $65 \pm 4$ gray-scale units, respectively. Non-operative contralateral shoulders had an average brightness value of $95 \pm 6$ gray-scale units. Representative images from each treatment and control group can be seen in Figure 4.

The area of new fibrocartilage formation was evaluated at the enthesis by determining the area of metachromasia of safranin $\mathrm{O}$-stained sections. Five specimens were analyzed for each treatment group. Contralateral shoulders of the acutely and chronically injured animals were not significantly different and were combined for analysis. The enthesis, after suture and construct repair of acute and chronic injuries, had significantly less fibrocartilage area than the native contralateral enthesis (Fig. 5). No significant difference in area of fibrocartilage formation was observed in acute injury suture or construct repairs. The area of fibrocartilage formation in the chronic injury group was significantly less in suture-repaired specimens compared with both construct-repaired specimens and unrepaired, contralateral shoulders. The total area of metachromasia, or area of fibrocartilage, for each treatment group was as follows: contralateral shoulders $\left(109,905 \pm 5095 \mu \mathrm{m}^{2}\right)$, acute suture repairs $\left(52,571 \pm 10,451 \mu \mathrm{m}^{2}\right)$, acute construct repairs $\left(67,063 \pm 11,455 \mu \mathrm{m}^{2}\right)$, chronic suture repairs $\left(11,272 \pm 6707 \mu \mathrm{m}^{2}\right)$, and chronic construct repairs $\left(40,432 \pm 7600 \mu \mathrm{m}^{2}\right)$ (Fig. 5). Representative 

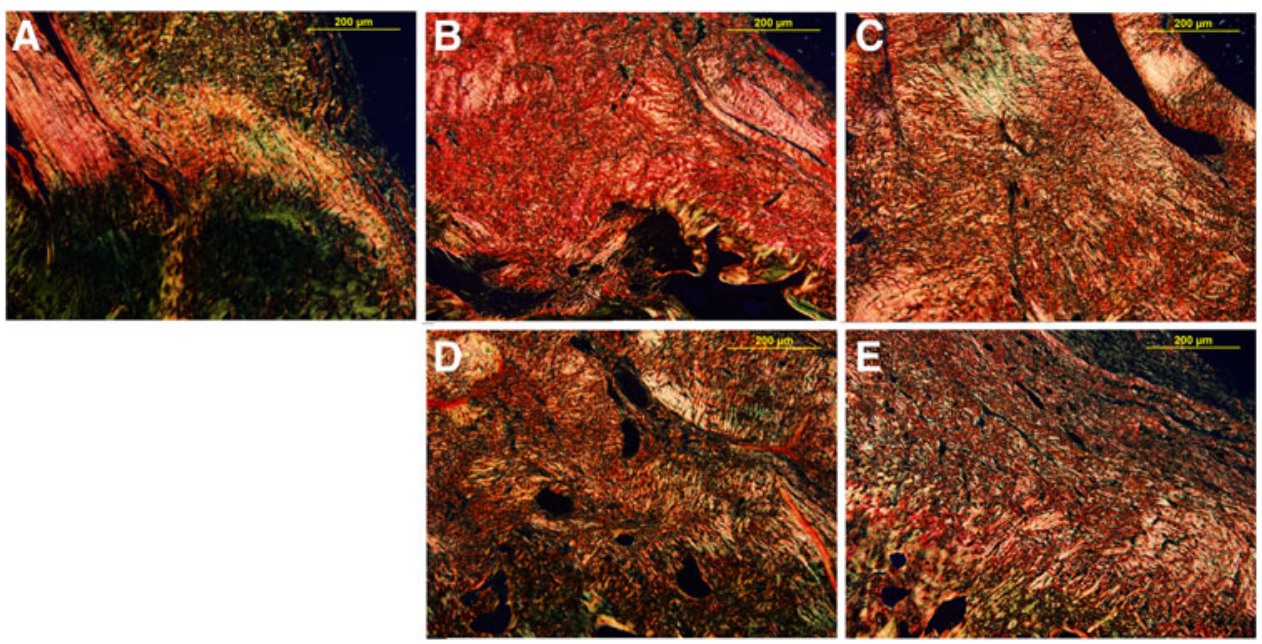

FIG. 4. Representative collagen organization of enthesis. (A) contralateral shoulder, (B) acute suture repair, (C) acute construct repair, (D) chronic suture repair, and (E) chronic construct repair. Images are of picrosirius red-stained sections under polarized light and imaged at $\times 40$ magnification.

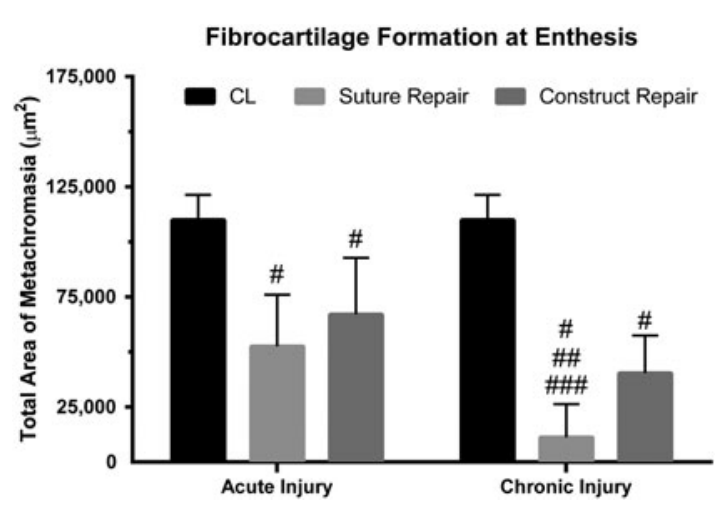

FIG. 5. Fibrocartilage formation at the enthesis after acute and chronic injury repair. Suture repair of chronically injured shoulders had significantly less fibrocartilage formation compared with both contralateral shoulder and construct repaired specimens. No significant differences were observed between acute and chronic injury groups.

(\#) Denotes statistical significance compared with $\mathrm{CL}$ shoulder $(p<0.05)$. (\#\#) Denotes statistical significance compared with acute suture repair $(p<0.05)$. (\#\#\#) Denotes statistical significance compared with chronic construct repair $(p<0.05)$. Data are shown as mean \pm SE for each group. images from each treatment and control group can be seen in Figure 6.

$\mathrm{H} \& \mathrm{E}$ staining of the tendon-bone interface allowed for qualitative evaluation of the entheses repair. Acute and chronic tendon injuries repaired with suture had less area of fibrocartilage formation and fewer organized collagen fibers than both construct-repaired and contralateral shoulders. The attachment of tendon to bone utilizing our tissue constructs resembled a more organized, native appearance. Suture-repaired shoulders in both acute and chronic injury models had a more abrupt, fibrous interface at the tendonbone transition site, especially in suture-repaired specimens of chronic injuries. Representative images from each treatment and control group can be seen in Figure 7 . There was no significant difference in the regeneration of the bone in the bone tunnel after repair with a construct or suture.

\section{Discussion}

Tissue engineering to improve the architecture and structural integrity of the regenerated enthesis after $\mathrm{RC}$ repair offers future promise to improve outcomes in patients. Engineered tendon constructs were used in this study based on their ability to specifically regenerate the enthesis in an ACL repair model. ${ }^{14}$ The objectives of this current study were to evaluate the efficacy of our scaffold-less tissue-engineered tendon construct 

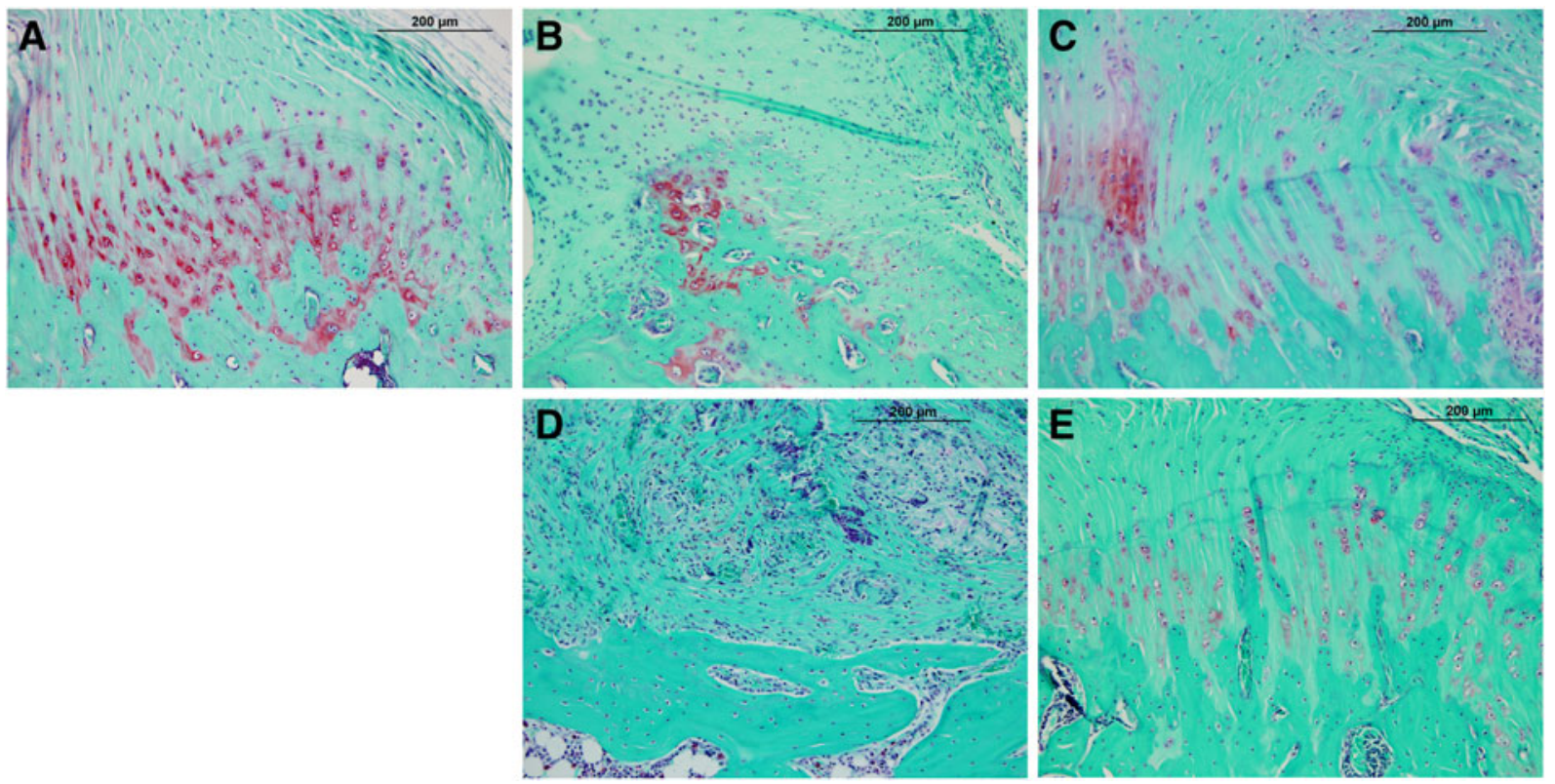

FIG. 6. Representative fibrocartilage formation at enthesis. Representative images of safranin $O$ stained (A) contralateral, (B) acute suture repair, (C) acute construct repair, (D) chronic suture repair, and (E) chronic construct repair sections. Area of metachromasia (depicted as red) in each image was outlined for semi quantitative analysis. Images are shown at $\times 40$ magnification.
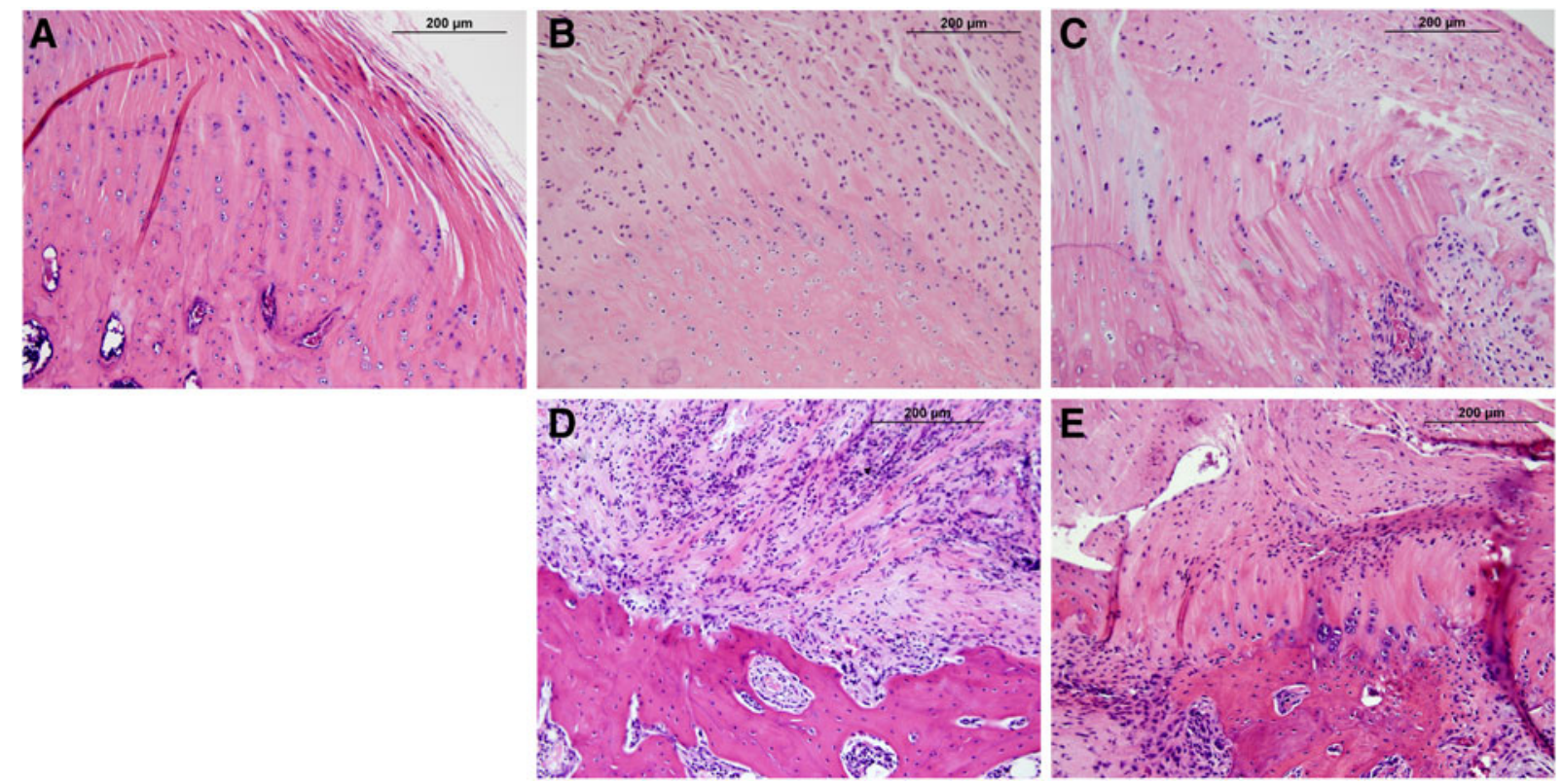

FIG. 7. Representative structure at enthesis. Representative images of H\&E stained (A) contralateral, (B) acute suture repair, (C) acute construct repair, (D) chronic suture repair, and (E) chronic construct repair sections. Suture-repaired shoulders were more disorganized and fibrous than contralateral- or construct-repaired shoulders. Images are shown at $\times 40$ magnification. 
to regenerate the enthesis of the supraspinatus tendon after full-thickness tears in an acute and chronic $\mathrm{RC}$ injury model in a rat. Furthermore, we tested the hypothesis that RC repair utilizing our scaffold-less tendon construct will improve the regeneration of the enthesis, improve collagen organization and fibrocartilage formation compared with suture fixation alone.

In the acute injury repair model, our results demonstrate that there were positive effects with the utilization of our scaffold-less tendon construct. Although no significant differences in biomechanical integrity were observed, tissue failure occurred more frequently at the enthesis in suture-repaired, acutely injured shoulders. Histologically, qualitative differences in enthesis morphology revealed more native histological architecture by 8 weeks post-operatively. However, no quantitative differences were observed in enthesis structure. Although no overall difference in the total proteoglycan content was observed between the groups (Fig. 6), there were areas of differing densities of proteoglycans. Han et. al have shown that micro-domains of proteoglycan density can have significant effects on micromechanics and, in turn, effects on the native tissue biomechanics. ${ }^{20}$ It could be that this variation in the density observed in the enthesis region of the different repair modalities explains the overall variation in tissue mechanics.

In tendons with chronic injury, repairs utilizing our tissue-engineered tendon construct resulted in a more native-like enthesis. Despite similar biomechanical properties with load-to-failure testing, suture-repaired specimens of chronic injuries failed more frequently at enthesis. However, the structure enthesis repaired with a tendon construct appeared qualitatively more native like in appearance and quantitatively more fibrocartilage was observed at the enthesis compared with suture repairs.

The formation of fibrocartilage at enthesis has an important role in the force transmission and energy dissipation between two tissues with vastly different properties. This complex tendon-bone interface is seldom recreated with current suture fixation techniques and often results in repair failure. The healing of the enthesis after repair is influenced by mechanical cues. In separate studies performed by Galatz et al., and Hettrich et al., complete removal of load after repair via immobilization and botulinum toxin application to supraspinatus muscles resulted in decreased structural properties and decreased
ECM production at the tendon-bone interface. ${ }^{21,22}$ As such, suture techniques that rely on rigid mechanical fixation of tendon to bone may impede signals that may be necessary for proper enthesis regeneration, resulting in a more fibrous tissue interface. The unique ability of our initially compliant tendon construct to sense and respond to local mechanical cues and biological factors has led to improved structure and fibrocartilage formation at the enthesis.

The biological composition of our graft may also contribute to repair of enthesis. Recent research has shown that application of MSCs, with or without the addition of tendon growth factors, can enhance tendon to bone healing. ${ }^{23}$ Our constructs are fabricated from MSCs and driven toward tendon lineage, via the addition of previously described growth factors. As such, enthesis regeneration at a repair site may be due to the unique cellular and molecular signals provided by the cells of our construct that are necessary for facilitating the proper host-mediated response. Alternately, cells within the implanted construct may be actively forming the newly regenerated enthesis in vivo. Current studies are underway to evaluate the origin of cells involved in the regeneration of the enthesis tissue.

Although our tendon construct is more successful at regenerating the enthesis than current suture techniques, the excessively high strains at the site of repair may limit repair outcomes. High tensile loads, especially in chronically retracted tendons, can lead to elongation of the tendon, reducing overall muscle length and compromising shoulder function. Though the length of tendon after repair was not evaluated in this study, gross morphological changes of the supraspinatus tendon and muscle were not observed. Nonetheless, the primary benefit of our construct may be its initial compliance and the ability to remodel its structural properties over time to match the mechanical properties that are necessary to stabilize and functionalize the shoulder. In addition, initial load sharing at the repair site utilizing our construct as a biological in-lay in conjunction with suture repair may enhance repair outcomes.

There were several limitations to this study. Mechanical testing evaluated stiffness and load to failure of a single test. Cyclic loading, a more biologically representative measure, was not performed. Furthermore, material properties utilizing tissue-level strains of the repaired tissue were not evaluated due to 
technical difficulties and may explain the lack of significant differences in mechanics, despite histological differences in tissue composition and location of failure. Assessment of the material properties of the regenerated tissue (i.e., moduli) utilizing tissuelevel strains, rather than only geometric properties (stiffness and load at failure) of the tissue, may better evaluate the quality of the regenerated tissue independent of size. Thus, the creation of a chronic supraspinatus injury in the rat may not fully represent the human condition. Healing in the rodent model is relatively robust and may not simulate the difficulties in healing and the structural failure after the repair of full-thickness tears observed in human patients. In addition, as this study focused specifically on enthesis regeneration, important components of RC injury specific to the tendon mid-substance and muscle such as muscle atrophy, fatty infiltration, quality of tendon, etc. were not evaluated.

In conclusion, the repair of acute full-thickness supraspinatus injuries in a rat model with engineered tendon constructs resulted in the regeneration of a more native-like enthesis but was not significantly different biomechanically compared with suture techniques. The utilization of our scaffold-less tendon construct for repair of chronic RC injuries was found to reduce scar formation and increase the area of fibrocartilage formation at the enthesis compared with suture repairs. Further investigation is needed to optimize the ideal tissue properties and surgical techniques for encouraging maximum enthesis regeneration in vivo.

\section{Acknowledgments}

The authors would like to acknowledge the $U$ of $M$ Department of Orthopedic Surgery for funding this project as well as NIH/NIDCR Tissue Engineering and Regeneration Training Grant (DE007057).

\section{Author Contributions}

Smietana-fabrication of engineered ligament, biomechanics data collection and analysis, and article preparation; Moncada-Larrotiz-histology data collection and analysis and article editing; Arruda-biomechanics data analysis and article editing; Bedi-experimental design, data analysis, and article editing; Larkinexperimental design, data analysis, and article preparation and editing. All authors have read and approved the final submitted article.

\section{Author Disclosure Statement}

No competing financial interests exist.

\section{References}

1. Jain NB, Higgins LD, Losina E, et al. Epidemiology of musculoskeletal upper extremity ambulatory surgery in the United States. BMC Musculoskelet Disord. 2014;15:4

2. Mansat $\mathrm{P}, \mathrm{Cofield} \mathrm{RH}$, Kersten TE, et al. Complications of rotator cuff repair. Orthop Clin North Am. 1997;28:205-213.

3. Galatz LM, Ball CM, Teefey SA, et al. The outcome and repair integrity of completely arthroscopically repaired large and massive rotator cuff tears. J Bone Joint Surg Am. 2004;86-A:219-224.

4. Zumstein $M A$, Jost $B$, Hempel J, et al. The clinical and structural long-term results of open repair of massive tears of the rotator cuff. J Bone Joint Surg Am. 2008;90:2423-2431.

5. Gumucio JP, Korn MA, Saripalli AL, et al. Aging-associated exacerbation in fatty degeneration and infiltration after rotator cuff tear. J Shoulder Elbow Surg. 2014;23:99-108.

6. Goutallier D, Postel JM, Gleyze P, et al. Influence of cuff muscle fatty degeneration on anatomic and functional outcomes after simple suture of full-thickness tears. J Shoulder Elbow Surg. 2003;12:550554.

7. Cofield RH, Parvizi J, Hoffmeyer PJ, et al. Surgical repair of chronic rotator cuff tears. A prospective long-term study. J Bone Joint Surg Am. 2001;83A:71-77.

8. Burkhart SS, Johnson TC, Wirth MA, et al. Cyclic loading of transosseous rotator cuff repairs: tension overload as a possible cause of failure. Arthroscopy. 1997;13:172-176.

9. Thomopoulos, S, Williams GR, Soslowsky LJ. Tendon to bone healing: differences in biomechanical, structural, and compositional properties due to a range of activity levels. J Biomech Eng. 2003;125: 106-113.

10. Derwin KA, Baker AR, Spragg RK, et al. Commercial extracellular matrix scaffolds for rotator cuff tendon repair. Biomechanical, biochemical, and cellular properties. J Bone Joint Surg Am. 2006;88: 2665-2672.

11. Zhang X, Bogdanowicz D, Erisken C, et al. Biomimetic scaffold design for functional and integrative tendon repair. J Shoulder Elbow Surg. 2012;21:266-277.

12. Smith L, Xia Y, Galatz LM, et al. Tissue-engineering strategies for the tendon/ligament-to-bone insertion. Connect Tissue Res. 2012;53: 95-105.

13. Montgomery SR, Petrigliano FA, Gamradt SC. Biologic augmentation of rotator cuff repair. Curr Rev Musculoskelet Med. 2011;4: 221-230.

14. Ma J, Smietana MJ, Kostrominova TY, et al. Three-dimensional engineered bone-ligament-bone constructs for anterior cruciate ligament replacement. Tissue Eng Part A. 2012;18:103-116.

15. Ma J, Goble K, Smietana M, et al. Morphological and functional characteristics of three-dimensional engineered bone-ligamentbone constructs following implantation. J Biomech Eng. 2009;131:101017.

16. Carpenter JE, Thomopoulos S, Flanagan CL, et al. Rotator cuff defect healing: a biomechanical and histologic analysis in an animal model. J Shoulder Elbow Surg. 1998;7:599-605.

17. Smietana MJ, Syed-Picard FN, Ma J, et al. The effect of implantation on scaffoldless three-dimensional engineered bone constructs. In Vitro Cell Dev Biol Anim. 2009;45:512-522.

18. Kovacevic D, Fox AJ, Bedi A, et al. Calcium-phosphate matrix with or without TGF-beta3 improves tendon-bone healing after rotator cuff repair. Am J Sports Med. 2011;39:811-819.

19. Cohen DB, Kawamura S, Ehteshami JR, et al. Indomethacin and celecoxib impair rotator cuff tendon-to-bone healing. Am J Sports Med. 2006:34:362-369.

20. Han WM, Heo SJ, Driscoll TP, et al. Microstructural heterogeneity directs micromechanics and mechanobiology in native and engineered fibrocartilage. Nat Mater. 2016;15:477-484.

21. Hettrich CM, Rodeo SA, Hannafin JA, et al. The effect of muscle paralysis using Botox on the healing of tendon to bone in a rat model. J Shoulder Elbow Surg. 2011;20:688-697. 
22. Galatz LM, Charlton N, Das R, et al. Complete removal of load is detrimental to rotator cuff healing. J Shoulder Elbow Surg. 2009;18: 669-675.

23. Gulotta LV, Kovacevic D, Packer JD, et al. Bone marrow-derived mesenchymal stem cells transduced with scleraxis improve rotator cuff healing in a rat model. Am J Sports Med. 2011;39:12821289.

Cite this article as: Smietana MJ, Moncada-Larrotiz $P$, Arruda EM, Bedi A, Larkin LM (2017) Tissue engineered tendon for enthesis regeneration in a rat rotator cuff model, BioResearch Open Access 6:1, 47-57, DOI: 10.1089/biores.2016.0042.

\section{Abbreviations Used}

$\mathrm{ACL}=$ anterior cruciate ligament ANOVA $=$ analysis of variance

$\mathrm{CL}=$ contralateral

$\mathrm{DM}=$ differentiation medium

DMEM $=$ Dulbecco's modified Eagle's medium

$E C M=$ extracellular matrix

$\mathrm{FBS}=$ fetal bovine serum

$\mathrm{GM}=$ growth medium

$\mathrm{H} \& \mathrm{E}=$ hematoxylin and eosin

MSC $=$ mesenchymal stem cells

$\mathrm{RC}=$ rotator cuff

\section{Publish in BioResearch Open Access}
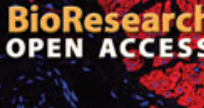

(c)

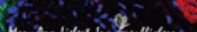

- Broad coverage of biomedical research

- Immediate, unrestricted online access

- Rigorous peer review

- Compliance with open access mandates

- Authors retain copyright

- Highly indexed

- Targeted email marketing

liebertpub.com/biores 